\title{
Physical Therapy and Functional Motor Recovery in Patient with Guillain-Barré Syndrome - Case Report
}

\author{
Antoaneta Dimitrova, PhD \\ Department of Physiotherapy and Rehabilitation, \\ National Sports Academy "V. Levski”, Sofia, Bulgaria \\ Nikolay Izov, PhD \\ Department of Aquatic Sports, National Sports Academy \\ "V. Levski", Sofia, Bulgaria \\ Ivan Maznev, PhD \\ Department of Sports Medicine, National Sports Academy \\ "V. Levski", Sofia, Bulgaria \\ Kristin Grigorova-Petrova, PhD \\ Daniela Lubenova, PhD \\ Department of Physiotherapy and Rehabilitation, \\ National Sports Academy "V. Levski", Sofia, Bulgaria \\ Dance Vasileva, PhD \\ Faculty of Medical Sciences, Goce Delchev University, \\ Shtip, Republic of Macedonia
}

Doi: 10.19044/esj.2017.v13n33p11 URL:http://dx.doi.org/10.19044/esj.2017.v13n33p11

\begin{abstract}
Introduction: Guillain-Barré syndrome (GBS) is an autoimmune disease with sudden onset and progressive impairment of the peripheral nerves. Aim: To study the influence of applied physical therapy (PT) on functional recovery in patient with a prolonged complicated course of GBS, Landry ascending paralysis and assisted breathing and 4 months in-hospital stay. Case report: The study was conducted with a 34-year-old woman in subacute stage of GBS, over the course of 2 months in the patient's home, after discharge. PT sessions were performed 3-4 times per week for 1-hour duration, moderate intensity, without reaching fatigue. Correct positioning in bed, passive-active exercises, analytical exercises, exercises for strength of abdominal muscles, breathing exercises, training balance and coordination of sitting and standing, and massage were applied. PT included correct positioning in bed, passive-active exercises, analytical exercises, exercises for strength of abdominal muscles, breathing exercises, training balance and coordination of sitting and standing, and massage. Tests for bed mobility and
\end{abstract}


transfers, Five Times Sit-To-Stand Test (FTSTS) and 10 Meters Walking Test (10MW) were applied twice in order to monitor the effect of PT.

Results and Discussion: The results showed an improvement in the speed of patient's response for transfers. Time for performing FTSTS decreased 33 sec. Improvement in bed mobility, walking speed and cadence are also observed. Conclusion: After two months of treatment functional mobility of the patient was improved.

Keywords: Guillain-Barré Syndrome, physical therapy, functional mobility

\section{Introduction}

Acute inflammatory demyelinating polyradiculopathy (Guillain-Barré Syndrome - GBS) is an autoimmune disease characterized by a sudden onset and progressive involvement of the nerve roots and peripheral nerves of the extremities. The first symptoms are sensory disturbances, and the leading symptom is the progressive symmetrical muscle weakness. It ranges from mild weakness to severe palsy of the four limbs, including the facial, respiratory, and swallowing muscles. The progression of the disease varies between one and four weeks, and in half of the patients, reversal was observed within two weeks. Approximately 10\% - 20\% of the patients develop respiratory failure. The mortality and disease chronification are about $2 \%-5 \%$. The acute phase is followed by a period of gradual recovery, usually over periods ranging from several months to two years. Diagnosis of the disease is based on a clinical, electromyographic, and laboratory criteria. Other diseases were excluded such as acute and subacute polyneuropathies, botulism and porphyria, basilar artery thrombosis, myelitis, spinal cord compression, AIDS infection, and others. Contemporary treatment includes administering high doses of immunoglobulins. Combination therapy with plasmapheresis and immunoglobulins is also used. Complete recovery has been achieved in over $70 \%$ of the patients with proper treatment. GBS has a serious impact on the patient quality of life, and in some cases leads to disability (Velcheva, 2015; Shotekov, 2002). The treatment and rehabilitation of GBS patients necessitates the use of serious medical-social events, which lead to improvement of their lifestyle, and emotional and psychological adaptation. The therapy is complex and requires a multidisciplinary team of specialists to produce an individual program that is maximally tailored to the patients' condition and their functional and motor possibilities. Its main focus is the application of tools to improve selfmanagement and independence in patients (Lubenova, 2011; Karaneshev, 1991).

Physiotherapy is a method that uses different forms of motor activity for functional recovery and maintenance of the functions or preventing the 
hypokinesia multiple effects. (Lubenova \& Titianova, 2012; Petkov, 2008). It is crucial to apply physiotherapeutic procedures as early as possible at a clinical stage, in order to prevent the following complications: deep vein thrombosis, dysautonomic disorders such as orthostatic hypotension due to prolonged immobilization, respiratory disturbances, respiratory muscle weakness, pain and fatigue (Davidson, 2009; Khan, 2004).

The serious consequences of GBS and the limited number of studies in this field are a well-founded reason for conducting in-depth research to find and apply an adequate physiotherapeutic approach.

\section{Case presentation Subject}

The monitored patient is a 34-year-old woman who entered a neurological clinic diagnosed with GBS. During the three-month in-hospital stay, she was admitted to an intensive care unit twice, on a mechanical ventilator controlled respiration with progressive respiratory insufficiency, due to the development of Landry type ascending paralysis.

\section{Outcome measures}

The functional research methods that were used were assessment of bed mobility (the time for turning from supine to the left/right side, from supine to sitting, from supine to standing was taken into consideration), 10Meter Walk Test (10MW), cadence during 10MW, and Five Times Sit-toStand Test (FTSTS). The first study was conducted two weeks after discharge from the hospital, and the second study was conducted two months after the hospitalization and applied rehabilitation at home.

The 10MW test was applied standardly and provided information about the walking speed at a short distance (Bohannon, 1997).

The FTSTS is used for functional assessment of the lower limb strength and also in various groups of patients with multiple diseases. The test is suitable for assessment of the neurological diseases (Bohannon, 2006; Dimitrova, 2008).

\section{Intervention}

During the hospital stay, individual physiotherapeutic procedures were performed daily, according to the patient's condition. After hospital discharge, the patient performed active rehabilitation sessions at home for the duration of two months: three times a week physiotherapist-supervised active training interventions supported by daily exercising alone after given physiothertherapist instructions. The treatment protocol included functional exercises with active patient participation based on her needs and optimizing residual motor function. The program progressed from active range of 
motion exercises to resisted functional exercises. Sessions typically lasted 60 minutes. Similar protocol was previously described to improve everyday functioning (i.e., domestic and community tasks to improve "activity"). Exercises prescribed started with low repetitions (usually for 5-10 repetitions) and short, frequent bouts of exercises corresponding to patient's muscle strength (Khan, 2011).

Proper positioning and postisometric relaxation to restore muscle imbalance and muscle tone were used. For pain reduction massage and stretching techniques were applied. It have been proven that they stimulate the proprioceptive sensation, improve circulation and reduce the pain. A slow and painless performance was recommended (Meythaler, 1997).

A sensory training with various stimuli for surface and shape (by sensing tools with a round or a cylindrical shape), in order to stimulate the proprioceptors and exteroceptors and to improve the different types of senses, was performed.

To improve voluntary movements or muscle contractions, proprioceptive neuromuscular facilitation and stimulation techniques were included.

Resistance exercises (manual, Thera-band, weights), were applied as an analytical training on the paretic muscles of the upper and lower extremities for a favorable influence on the motor disorders.

The resistance exercises had a positive influence on the motor disturbances, improving the blood supply to the tissues which in turn provided better oxygen and metabolic needs.

Cyclical exercises (walking on a flat surface, walking up and down on different slopes, climbing and descending stairs) were also performed.

In order to improve normal postural mechanisms and balance reactions in walking were included exercises influenced equilibrium and balance reactions with change of support area, weight shifting, gravity line crossing, walking with eyes closed, and walking with movement retention in the swing phase.

The impaired equilibrium and balance capabilities, as a result of sensory disturbances, were basically compensated visually and impaired biomechanics balance factors (center of gravity, line of gravity, base of support, and levels of stability) due to muscle weakness in the distal parts of the lower limbs, reduced range of motion in the ankle joints and pain. Based on the mentioned static and dynamic balance disturbances, the principal recommendations for physiotherapeutic influence on the polyneuropathy sensory ataxia aimed to improve the proprioception by excluding or destabilizing the visual analyzer while standing on a hard surface.

Volitional movement control was stimulated by coordination exercises - locomotion with closed eyes, movement of the same upper and 
lower extremities in different directions, movement of the opposite upper and lower limbs in different directions, keeping the movement under command.

The intensity was consistent with the functional capabilities of the cardiovascular system, which required a three-fold heart rate and arterial blood pressure measurement during the session performance, as well as the appearance of rapid fatigue in GBS patient (Arsenault, 2016, El Mhandi, 2007). Exercise intensity was closely monitored and the PT was terminated before the patient reported fatigue (Meythaler, 2007, Garssen, 2004, Khan \& Amatya, 2012).

Our goal was to restore the functional status and self-management by normalizing the muscle imbalance and muscle tone; stimulation of the proprioceptors and exteroceptors for improving the senses, improving the normal postural mechanisms, balancing walking responses, and stimulating the volitional movement control.

\section{Results and discussion}

The data obtained in this study was traced twice and it is presented in table 1.

Table 1. Changes in functional motor activity before and after treatment

\begin{tabular}{cccc}
\hline Test & $\mathrm{X}_{1}$ & $\mathrm{X}_{2}$ & $\mathrm{X}_{1}-\mathrm{X}_{2}$ \\
\hline $\begin{array}{c}\text { Bed mobility: turning from supine } \\
\text { to the left side (sec) }\end{array}$ & 3 & 1,2 & 1,8 \\
Bed mobility: turning from supine & & & 3,2 \\
to the right side (sec) & 4,9 & 1,7 & 0,4 \\
Lying to sitting (sec) & 3 & 2,6 & 2,2 \\
Sitting to standing (sec) & 7,8 & 5,6 & 33 \\
FTSTS (sec) & 49 & 16 & 23,8 \\
10 metres walking (sec) & 40 & 16,2 & 0.35 \\
10 metres walking (m/sec) & 0.25 & 0.6 & 12 \\
Cadence for 10 metres walking & & 23 & \\
(number of steps) & 35 & & \\
\hline
\end{tabular}

$\mathrm{X}_{1}$ - initial assessment; $\mathrm{X}_{2}$ - final assessment; FTSTS - Five Times Sit To Stan

The patient's program improved bed mobility, sitting balance, and saved transfer skills to enable progressive ambulation and gait training.

In the transfer assessment (transition from supine to the left/right side, from supine to sitting, from supine to standing) rate improvement of the patient's reactions, as well as the "Five times sit-to-stand" test (with $33 \mathrm{sec}$ ) were observed. The results showed improvements when comparing baseline and post-training in walking abilities. Time for walking $10 \mathrm{~m}$ distance decreased with $23.8 \mathrm{sec}$, and in the cadence in $10 \mathrm{MW}$, the number of steps decreased to 12 in the second study. The correctly applied physiotherapeutic methodology was mostly individually tailored. Before conducting PT 
sessions, clarification of the patient's functional potential was necessary. There should be an optimal load depending on the clinical and functional condition of the patient. If the load is mild (sub-threshold irritation), there is no adequate physiological and therapeutic effect respectively, and vice versa. If the load is excessive, there is a rapid onset of unwanted fatigue and nervous tension or undesirable reactions in an organism. Physiotherapy should be applied systematically and regularly, to accumulate the effect and lead to changes in the body functional and motor reactions. Systemic application of PT requires the creation of a specific plan that includes the purpose, the tasks, and the tools. Task solving should have a certain sequence according to the stage of the disease. The duration of PT application also depends on the type and severity of the disease. Moreover, physiotherapy is an important part of optimal recovery in patients with GBS. The physiotherapeutic goal is to improve the psychosomatic condition and quality of life of patients with Guillain-Barré syndrome (Davidson, 2009). In order to prevent complications in the respiratory and cardiovascular system, as a result of prolonged immobilization, it is recommended that PT be applied as early as possible at a clinical stage. Because of the rapid and easy fatigue of the patients, there should be presence of exercise distraction during the PT treatment and frequent breaks. It is recommended that the following be included: breathing exercises, massage, training in safe transfer techniques, a passive verticalisation, use of ankle orthosis for preventing foot contractures, passive exercises to maintain the range of motion in the joints, exercises with adequate resistance to the lower extremities, such as preparation for walking and others. Great importance is placed on learning to walk independently (Khan, 2004).

At each stage, the patient was prepared to walk. The following were also performed: changing and maintaining body position during functional mobility, starting with a self-turning/rolling over in bed during the acute stage, exercises on kneeling all fours (quadruped position), moving from sitting to standing, equilibrium and balance exercises, using walking aids, correct gait-training, and self-guided walking. PT should be aimed at solving specific tasks for the relevant period and taking into account the clinical course of the disease (Lubenova, 2011; Karaneshev, 1991; Lubenova \& Titianova, 2012; Petkov, 2008).

The bed functional mobility and transfers assessment did not focus on movement quality used to accomplish the tasks, but provided information about patient's reaction and functional mobility needed for daily personal care including bathing and toileting. Safe transfer and bed mobility are crucial for functional independence in daily life. We suppose that improvement of the transfers were due to the systematic application of PT, 
which included exercises with frequent change of body position (Lubenova, 2011; Lubenova \& Titianova, 2012).

The improvement of lower extremity muscle strength is a major step toward achieving patient independence. Sit to stand training aims to establish coordination and consistency of movements between the trunk and lower limbs. Therefore, in rising to standing up, the correct position of the body and the speed of movement are essential. Timing, speed of trunk movement and correct starting body positioning are important for effective, energy conserving transition. If the trunk movement speed is decreased, then it will be a greater loading of the lower extremities Thus, through the purposeful training of rising to standing up, the coordination and consistency of movements between the trunk and lower limbs were influenced and improved, probably due to the applied resistance exercises in the PT sessions (Petkov, 2008, Davidson, 2009).

Walking speed indicates the patient's independence for daily living activities, not just at home but also in situations requiring a response, such as a pedestrian crossing (street). The 10MW test reports short-distance velocity, i.e. indicators, such as endurance or walking on a different surfaces, are not evaluated. In the present study, the patient's velocity increased from an initial velocity of $0.25 \mathrm{~m} / \mathrm{sec}$ to $0.6 \mathrm{~m} / \mathrm{sec}$. When comparing the speed of movement and the possibility of functional activities, Perry et al. (1995) reported that a velocity of less than $0.4 \mathrm{~m} / \mathrm{sec}$ is used by patients walking only in a home environment; from $0.4 \mathrm{~m} / \mathrm{sec}$ to $0.8 \mathrm{~m} / \mathrm{sec}$, by patients with some degree of movement freedom and in other environment, and over $0.8 \mathrm{~m} / \mathrm{sec}$ is used by patients independent in their moving, performing functional activities of daily life and participation in society (Perry, 1995).

\section{Conclusion}

We would like to recommend our methodology of therapeutic exercises according the stage of the disease, because after the applied physiotherapy, our patient experienced a functional improvement of the paretic extremities, which was associated with gradual muscular strengthening and improved balance and gait which was determined by reducing the number of steps and increasing the movement speed. Improvement in walking speed was sufficient to allow the patient to be selfconfident and independent in outdoor activities and in the same time intermittent follow-up is still necessary.

\section{References:}

1. Velcheva I, Genova K, Dimova R, Lubenova D, Popov P, Stamenov B. (2015) Ed. by Ekaterina Titianova. Textbook on Nervous Diseases. General Neurology. University "Sv. Kliment Okhridski", Sofia. 
2. Shotekov P. (2002) Textbook of Neurology. MI "ARSO", Sofia

3. Lubenova D. (2011) Kinesitherapy of neurogical and psychiatric diseases. Betaprint-Petrovi i Sie, Sofia

4. Karaneshev G. (1991) General theory and method of kinesitherapy. Medicine and gymnastics, Sofia.

5. Lubenova D, Titanova E. (2012) Principles of modern neurorehabilitation. Neuroscience and cerebral hemodynamics, 8(1): 45-55.

6. Petkov I. (2008) Basics in kinesitherapy. Means and specialized techniques in kinesitherapy. Tip-top Press, Sofia.

7. Davidson I, Wilson Ch, Walton T, Brissenden Sh. (2009) Physiotherapy and Guillain-Barré syndrome: results of a national survey. Physiotherapy, 95:157-163

8. Khan F. (2004) Clinical practice: Rehabilitation in Guillian Barre syndrome. Australian Family Physician, 33(12): 1013-7

9. Bohannon R. (1997) Comfortable and maximum walking speed of adults aged 20-79 years: reference values and determinants. Age Ageing, 26 (1): 15-9.

10. Bohannon, R. (2006) Reference values for the five-repetition sit-tostand test: a descriptive meta-analysis of data from elders. Percept Mot Skills, 103(1): 215-222.

11. Dimitrova A, Simeonova A, Grigorova K, Stefanova D. (2008) The influence of kinesitherapy over dynamic balance in patients with ischemic stroke in vertebral basilar system. Trakia Journal of Science, 6 (2):80-83.

12. Khan F, Pallant J, Amatya B, Ng L, Gorelik A, Brand C. (2011) Outcomes of high- and low-intensity rehabilitation programme for persons in chronic phase after Guillain-Barré syndrome: a randomized controlled trial. J Rehabil Med, 43(7):638-46.

13. doi: 10.2340/16501977-0826.

14. Khan F, Ng L, Amatya B, Brand C, Turner-Stokes L. (2011) Multidisciplinary care for Guillain-Barré syndrome. Eur J Phys Rehabil Med, 47(4):607-12.

15. Meythaler J. (1997) Rehabilitation of Guillian-Barre syndrome. Review. Arch Phys Med Rehabil, 78:872-879

16. Arsenault N, Vincent P, Shen Yu , Bastien R, Sweeney A. (2016) Influence of Exercise on Patients with Guillain-Barré Syndrome: A Systematic Review. Physiother Can, 68(4):367-376.

17. doi: 10.3138/ptc.2015-58

18. El Mhandi L, Calmels P, Camdessanché J, et al. (2007) Muscle strength recovery in treated Guillain-Barré syndrome: a prospective 
study for the first 18 months after onset. Am J Phys Med Rehabil, 86(9):716-24. http://dx.doi.org/10.1097/PHM.0b013e31813e0b67

19. Garssen M, Bussmann J, Schmitz P, et al. (2004) Physical training and fatigue, fitness, and quality of life in Guillain-Barré syndrome and CIDP. Neurology, 63(12):2393-5. http://dx.doi.org/10.1212/01.WNL.0000148589.87107.9C.

20. Khan F, Amatya B. (2012) Rehabilitation interventions in patients with acute demyelinating inflammatory polyneuropathy: a systematic review. European Journal of Physical and Rehabilitation Medicine, 48(3):507-22

21. Perry J, Garrett M, Gronley J, Mulroy S. (1995) Classification of walking handicap in the stroke population. Stroke, 26(6):982-989. 